A study has been made of induction bridges used in low temperature physics.

In Part 1 the design of a mutual inductance bridge of the Hartshorn type is discussed. This design is based on a critical analysis of impurity effects of the different parts of the Hartshorn bridge. With this equipment frequencies up to $0.5 \mathrm{MHz}$ can be used. Two methods have been developed to examine the secondary signal. In one of these use has been made of $A D$ conversion techniques. In the other one, the secondary signal, produced by a superconducting sample, which is generally distorted, is analysed by using a Fourier expansion.

In Part 2 equipment is described which enables us to measure the phase and amplitude of the harmonics of the output signal of the bridge. For synchronous detection a reference signal of the same frequency of the harmonic of interest is required. This reference signal is generated from the input signal of the bridge by means of a digital frequency multiplier with programmable multiplication factor $N$.

In Part 3 some experimental results, showing the possibilities of the equipment, on some superconductors are presented.

\title{
Induction methods used in low temperature physics
}

\author{
L. J. M. van de Klundert, C. de Rooij, M. Caspari, and L. C. van der Marel
}

Many more or less successful attempts to investigate the behaviour of paramagnetic salts in alternating fields were made in the 1920s.

An excellent short survey of these is presented by Verstelle and Curtis. ${ }^{1}$ An important impetus to further development was the proposal of Debije and Giauque in 1926 that extremely low temperatures could be attained by adiabatic demagnetization. By using induction methods the magnetic temperature, defined by Curie's law, and in many cases a measure of the thermodynamic temperature, can be determined. The problem of establishing the magnetic moment in a changing magnetic field was important here and led to numerous experiments on an independent field of investigation, called paramagnetic relaxation. For temperature and relaxation measurements in the liquid hydrogen and helium ranges, as well as in the millidegree temperature range, frequent use is made of self-inductance and mutual inductance bridges. Reference is made here to the work of Casimir, ${ }^{2}$ De Klerk and Hudson, ${ }^{3}$ Erickson, ${ }^{4}$ Wiebes, ${ }^{5}$ Pillinger, ${ }^{6}$ Hoffman and Sapp, ${ }^{7}$ Van Dijk, ${ }^{8}$ Cremer, 9 De Vries and Livius, ${ }^{10}$ etc. Impurity effects of the different elements of the induction bridge did not have much effect on temperature measurements; restriction to the use of one frequency was possible and as a rule this frequency was so low that there were no problems with, for example, capacity effects of the coils. Only temperature effects of the coil system in which the material to be investigated was found, can give rise to difficulties. However, in case frequency effects are determined, self- and inter-capacitances of different parts of the measuring system may reduce the effects to be investigated.

The authors are in the Department of Technical Physics and Department of Electrical Engineering, Twente University of Technology, Enschede, The Netherlánds. Received 16 April 1975.
In the first part of this paper a critical analysis of these effects in a Hartshorn-type bridge is given; the choice of this type of bridge is discussed. Using the results of this analysis a mutual induction bridge, using toroidal windings both in the primary and secondary circuit, has been designed and built. With this bridge it is possible to extend the frequency range up to $1 / 2 \mathrm{MHz}$. The second part of this paper discusses the analysis of the signal, generated in the detection coils. Naturally this is a direct measurement of the property under investigation. In cases where the current in the primary circuit is sinusoidal it is generally assumed that the signal in the secondary circuit is also sinusoidal. If the sample in the coil system is a paramagnetic salt this assumption results from the supposition that the magnetic behaviour of a paramagnetic salt can be described with a time-independent complex susceptibility, the amplitude of the ac field being very small. If the sample is a metal this assumption is not violated at small amplitudes of the ac field. Induced eddy currents in cylindrical metals were described by Zenneck in $1902{ }^{11}$ who also made the local $B-H$ curve on a Braunian tube visible. If the sample is a superconductor the secondary signal cannot be described only by a single harmonic signal and a Fourier series must be used. ${ }^{12,13,14,15}$

In Part 2 electronic equipment, which enables the coefficients of this Fourier series to be determined separately, is described. In contrast to the detection procedure of compensating the signal in the measuring circuit with that of the bridge circuit, automatic detection is applied. This resulted in a considerable time saving during the measurements.

The paper is concluded with a presentation of some experiemental results on superconducting tin and niobium. 


\section{Part 1: Design of a mutual inductance bridge Choice and general description of the method}

When a sample of apparent differential susceptibility $\chi$ is placed in a coil with a pure self-inductance $L_{0}$, the selfinductance will change to

$$
L=L_{0}\left(1+q_{L} \chi\right)
$$

so

$$
\Delta L=L-L_{0}=L_{0} q_{\mathrm{L}} \chi \text { or } \chi=\frac{\Delta L}{q_{\mathrm{L}} L_{0}}
$$

where $q_{L}$ represents a filling factor.

This will give rise to an extra potential drop $\Delta U$ across the coil when the same current $I$ is fed into it

$$
\Delta U=j \omega L \Delta L=j \omega I L_{0} q_{\mathrm{L}} \chi
$$

or

$$
\frac{\Delta U}{j \omega I L_{0}}=q_{\mathrm{L}} \chi
$$

Imbedding the specimen into a pair of coils with a pure mutual inductance $M_{0},\left(M_{0}=k \sqrt{ }\left(L_{1} L_{2}\right), L_{1}\right.$ and $L_{2}$ being respectively the self-inductance of the primary and secondary coil and $k$ the coupling factor) will give rise to an extra induced voltage in the secondary coil amounting to

$$
\frac{\Delta U}{j \omega I M_{0}}=q_{\mathrm{M}} \chi
$$

$q_{\mathrm{M}}$ also represents a filling factor.

Equations 1 and 2 form the basis of the inductive methods which consists of measuring directly or by compensating $\Delta U$ in the bridge circuit. So two methods exist using either a self-inductance or a mutual inductance bridge. Both methods are limited in their frequency range by the fact that a self-inductor and, a fortiori, a mutual inductor is impure. In other words if we combine (1) and (2) to give $\Delta U=A \chi, A$ will no longer be a purely imaginary factor.

An extensive survey of ac bridge methods is given by Hague and Foord. ${ }^{16}$ As can be seen from (1) and (2) compensation can be obtained by either varying the value of compensating elements using one bridge current, or by adjusting the values and phases of at least two bridge currents using only a few fixed-value compensating elements. Both methods have their advantages. In the first method the disadvantage comes from the fact that the impurities of the various elements are different. The second method is limited by the difficulty in controlling the (possibly complex) ratio $\alpha$ of the currents.

A few successful examples illustrating both possibilities, not compiled by Hague and Foord, may be mentioned. In the first place the self-inductance bridge designed by De Vries, ${ }^{10}$ operating in a frequency range $200 \mathrm{~Hz}-1 \mathrm{MHz}$.

The ratio $\alpha$ of the bridge currents is here a real number. Pederson ${ }^{17}$ constructed a bridge with a purely imaginary $\alpha$ by applying a resonance technique. This enabled him to use frequencies up to $100 \mathrm{kHz}$, but now a low frequency limit is set by bringing the bridge into resonance.

The mutual inductance bridges have several advantages (see the end of this section) and all are discussed by Hartshorn.

In the original paper of Hartshorn, 18 two proposals for a mutual inductance bridge were made, one with two primary currents that are brought into phase by means of resistive elements, the impurities of which do not effect the mutual inductors, and a second, in which only one primary current is used. The latter is very well-known in adiabatic demagnetization and paramagnetic relaxation measurements. Various designs of a bridge of this type were published, among which those of De Klerk and Hudson ${ }^{3}$ and Erickson et al ${ }^{4}$ are often used, and will be discussed later.

Wiebes et al ${ }^{5}$ published a description of a two-branch mutual-inductance bridge in which the current is divided by resistors and capacitors. The use of series capacitors also limits the frequency range to a lowest frequency of about $100 \mathrm{~Hz}$, while the high frequency limit, because of the impurities, is $10^{4} \mathrm{~Hz}$.

A bridge with electronic current division was first discovered by Pillinger et al ${ }^{6}$ operating at a frequency of $150 \mathrm{~Hz}$.

Campbell 19 pointed out that an extra secondary or tertiary coil of only one turn can reduce the frequency dependence of self and mutual inductors considerably by short-circuiting this loop with an appropriate resistor. From his experiments it is seen that when the frequency dependence of the reactive part of the inductance is reduced, the frequency dependence of the resistive part is considerably increased. Therefore this method was not adopted in our final design.

In our laboratory two mutual inductance bridges of the Hartshorn type with only one primary current were constructed. The first one was designed using the work of De Klerk et al. 2,3 Extensive experimental research was carried out on the frequency limiting elements. The results will be described later in this paper. Using these results a new bridge was designed and constructed, and this bridge will also be discussed later.

The term 'apparent differential susceptibility' used above needs some explanation. The total magnetic moment of the sample, which may not be in phase with the external field, can arise

(a) from magnetic moments on a microscopic scale,

(b) from induced macroscopic currents, as in conductors and superconductors, or

(c) from a combination of these two effects.

It is well-known that, for example, superconductors under certain conditions behave as non-linear elements, that is to say the hysteresis loop is no longer an ellipse.

So it is convenient then to develop $m(t)$ in a Fourier series

$$
m(t)=h_{0} \mathrm{e}^{-j \omega t} \sum_{k=1}^{\infty} \chi_{k} \mathrm{e}^{-j(k-1) \omega t}
$$

with

$$
\chi_{k}=\chi_{k}^{\prime}+j \chi_{k}^{\prime \prime}
$$


To measure the value of $\chi_{k}^{\prime}$ and $\chi_{k}^{\prime \prime}$ properly a frequency multiplier is essential giving an output proportional to $\mathrm{e}^{-j k \omega t}$ in case the current fed into the primary circuit is represented by $I \mathrm{e}^{-j \omega t}$.

To determine the output signal of the secondary circuit of the bridge, two systems have been developed and used in our laboratory. The first system determines the $\chi_{k}^{\prime}$ and $\chi_{k}^{\prime \prime}$ values. The bridge signal is, after amplification, fed into two phase-sensitive detectors (PSD) in parallel which have a $\pi / 2$ relation to each other. The output of each PSD was fed into $x-y$ recorders for automatic detection. No corrections to the recorder plots are necessary, as will be discussed later. Depending on the pre-amplification factor, a relative accuracy of better than 1\% can be obtained. Each PSD is part of a lock-in amplifier which is synchronized by the frequency-multiplier unit, described in part 2.

A block diagram of the measuring circuit is given in Fig.1.

The second system has been recently developed; the main feature is the use of $\mathrm{AD}$ conversion techniques. Two signals are delivered by the bridge circuit: a reference signal and the signal generated in the secondary coil part of the bridge and giving information about the sample to be investigated. Both are registered in a memory ( $\mathrm{TR}=$ transient recorder $)$ by means of a trigger-reference-signal switch (TRS). This switch is activated in the following way. The positive zero crossing of the reference signal and an independent clock signal are indicated by short pulses; at the moment that both signals coincide the counter and the TR start-up. In this way a unique phase relation between the two bridge signals, which is essential for the interpretation of the measuring results, is achieved.

With the aid of an electronic switch, triggered by the counter (part of TRS), a switch is made from the reference signal, coming immediately from the bridge (length: for example, 256 words) to the measuring signal (for example, 768 words). If the memory has been filled the contents of it is, without deconversion, recorded on a paper tape. The TR can be activated in two ways: triggered by its own clock or triggered by an external pulse generator.

This measuring circuit is shown in Fig.2.

Two reasons made us decide to use a mutual inductance bridge rather than a self-inductance bridge. First, the amplitude of the disturbing field is only determined by the primary windings whereas the resulting signal is related to both primary and secondary coils, giving an extra degree of freedom. This is especially important when the signal is amplitude-dependent as, under certain circumstances, is the case with superconductors. Then it is possible also to get signals which can be easily detected at low amplitudes and frequencies by increasing the number of secondary turns.

A second advantage comes from the fact that it is necessary to eliminate coupling from the surroundings by using astatic pairs of coils. Now the change of inductance in the coils of such a pair, due to temperature variations and pressure effects, will add up in the case of self inductors and will almost cancel in the case of mutual inductors. Such changes are very difficult to calibrate.

\section{Frequency effects in detection coils}

A block diagram of the various elements of the bridge is given in Fig.3. This figure does relate to Fig.1 but the following considerations are also applicable to the system, shown

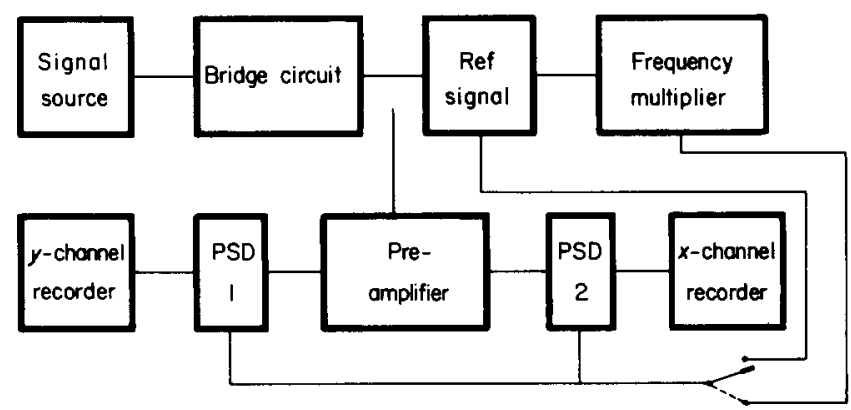

Fig. 1 Block diagram of the measuring circuit; first system

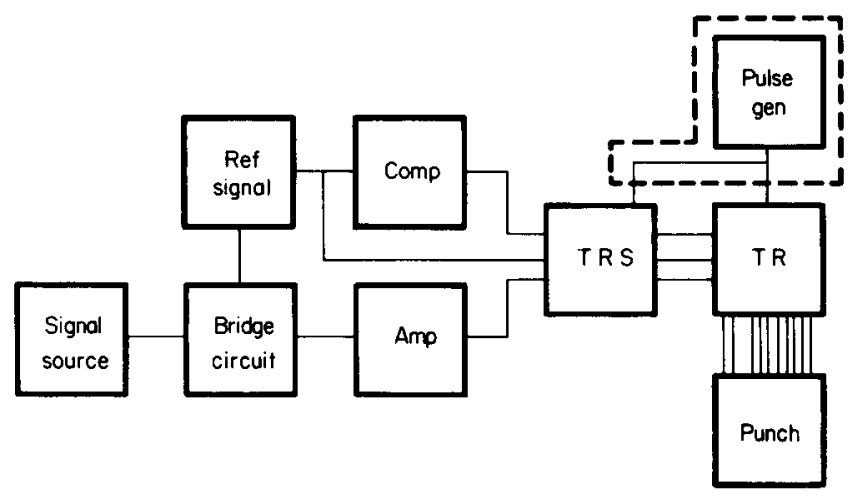

Fig.2 Block diagram of the measuring circuit; second system

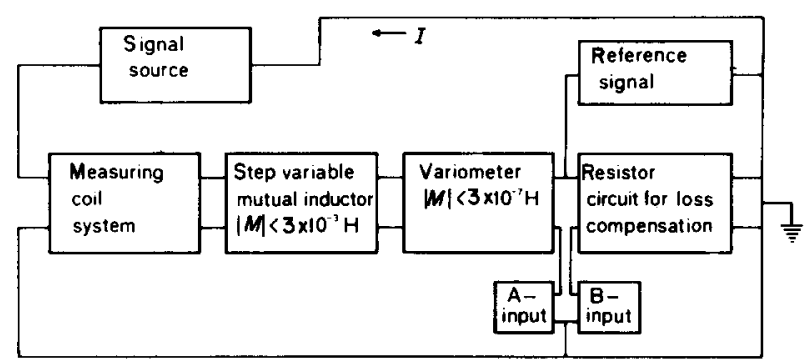

Fig.3 Block diagram of the bridge circuit

by Fig.2. We will now discuss the frequency dependence of these elements; because of the complexity of the problem a rigorous treatment is not attempted. The properties of impure mutual inductors have been extensively studied by Butterworth ${ }^{20}$ and Hartshorn, ${ }^{21}$ both theoretically and experimentally.

They have shown that the factors introducing impurities are:

1. Self- and inter-capacitances of the windings.

2. Eddy current losses in the wiring and inductive coupling with the surroundings.

3. Leakage and dielectric losses in the insulation.

4. Resistance inadvertently included in common with both windings when these are connected at a common point. This last point is in our case only of interest in the mutual inductors describing the coupling between secondary windings, because there is no common point of the primary and secondary circuits in the inductive part of the bridge.

The measuring coil system. It is obvious that the main effect is from the first two mentioned. To give an example we will discuss the effects on the measuring coil system (see Fig.3). 
It is well-known that the skin effect in the wiring can be considerably reduced by using thin high conductivity wire or litz wire. But since our measuring coil system is at liquid helium temperatures, it would not be sensible to use litz wire. All coils are wound from $0.07 \mathrm{~mm}$ silk covered copper wire. The skin depth at $5000 \mathrm{~Hz}$, taking $\sigma=6 \times 10^{9} \Omega^{-1} \mathrm{~m}^{-1}$, is approximately $0.13 \mathrm{~mm}$. Therefore no further attention is drawn to this point. The coupling with the surroundings can be eliminated by using astatic pairs for both the primary and the secondary coils. In the primary circuit this is achieved by using two concentric coils of equal length and radius $r_{1}$ and $r_{2}$ (see Fig.4).

The system now acts as an astatic pair when $N_{1} r_{1}{ }^{2}=$ $N_{2} r_{2}{ }^{2} ; N_{1}$ and $N_{2}$ being the number of turns of each winding. Since this becomes very important when a co-axial superconducting coil is also used to produce a static field, we shall come back to this point later.

The equivalent circuit describing the influence of self and of inter-capacities in a mutual inductor, is given in Fig.5a, according to Butterworth. ${ }^{20}$ In our arrangement the effects of the intercapacities from simplified calculations are small compared to that of the self-capacities of the multilayer coils. Therefore they are neglected in the scheme shown in Fig.5b, describing the measuring coil system. For simplicity the primary windings are considered as a single $R L C$ mesh.

A relation between the total induced voltate $\Delta U$ across the secondary windings and the bridge current can be obtained as described below.

Although this relation can be obtained with rather crude assumptions, it will still show the frequency limiting properties of such a coil system.

In the primary circuit the current through the coil $L_{\mathrm{p}}$ will be different in both phase and magnitude from the bridge current $I$.

Neglecting the small emf that may be induced due to the fact that the secondary windings do not balance exactly, the

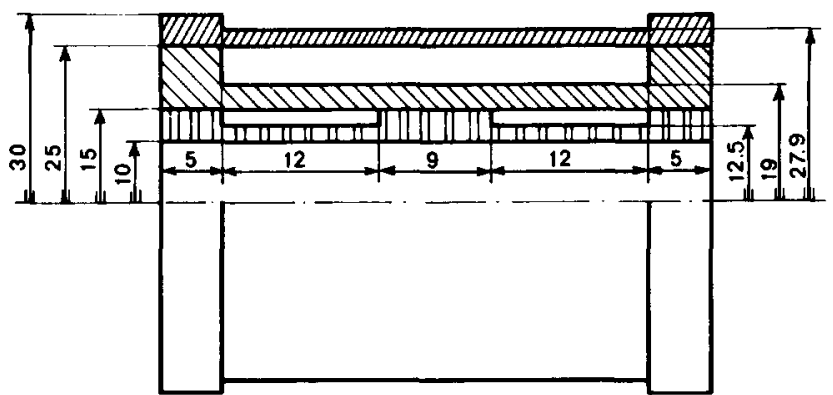

Fig.4 Cross-section of the measuring coil system

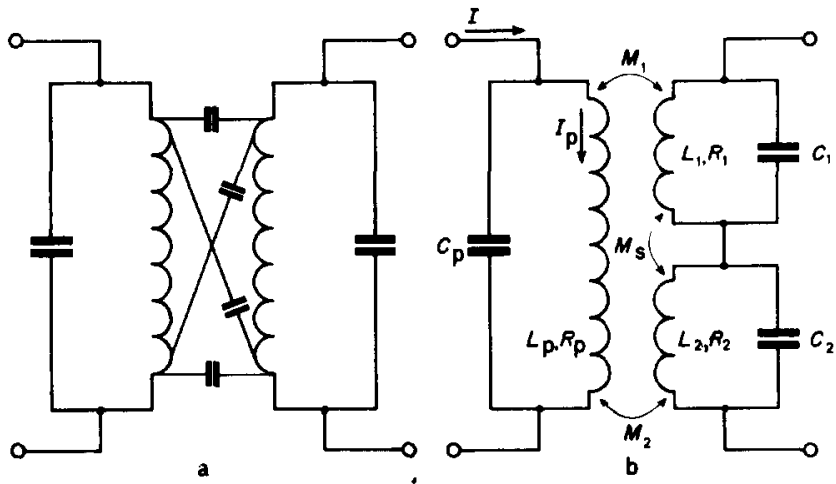

Fig.5 a - Equivalent circuit of the impure mutual inductor; $b$ - Simplified circuit of the measuring coil system following relation between $I$ and $I_{\mathrm{p}}$ holds

$$
I_{\mathrm{p}}=\frac{I}{1-\omega^{2} L_{\mathrm{p}} C_{\mathrm{p}}+j \omega R_{\mathrm{p}} C_{\mathrm{p}}}
$$

The secondary voltage $\Delta U$ can now be written

$$
\Delta U=\frac{I_{1}}{j \omega C_{1}}+\frac{I_{2}}{j \omega C_{2}}
$$

$I_{1}$ and $I_{2}$, the currents in the secondary meshes, can be solved from the equations expressing Kirchhoff's voltage law for the upper and lower secondary coil respectively.

$$
\begin{gathered}
\left(1-\omega^{2} L_{1} C_{1}+j \omega R_{1} C_{1}\right) I_{1}+\omega^{2} M_{\mathrm{s}} C_{1} I_{2} \\
=\omega^{2} M_{1} C_{1} I_{\mathrm{p}} \\
\begin{aligned}
\omega^{2} M_{\mathrm{s}} C_{2} I_{1} & +\left(1-\omega^{2} L_{2} C_{2}+j \omega R_{2} C_{2}\right) I_{2} \\
& =-\omega^{2} M_{2} C_{2} L_{\mathrm{p}}
\end{aligned}
\end{gathered}
$$

Great care was taken during the construction of the secondary coils to obtain almost identical properties. Therefore it is permissible to assume $R_{1}=R_{2}$ and $C_{1}=C_{2}$.

Equations $5 \mathrm{a}$ and $5 \mathrm{~b}$ may be solved readily, considering that the terms containing $M_{\mathrm{s}}$ are very small compared to the others and therefore can be omitted.

Retaining small differences $\Delta L=L_{2}-L_{1}$ and $\Delta M=M_{2}-M_{1}$, in the first-order approximation, (4) can be written

$$
\Delta U=j \omega I_{\mathrm{p}}\left(\frac{\Delta M}{N}+\omega^{2} M C \Delta L\right)
$$

with

$$
N=1-\omega^{2} L C+j \omega R C
$$

(the suffixes are now suppressed).

From (6) it can be seen that if $\Delta L$ and $\Delta M$ are zero, $\Delta U$ vanishes.

So far only an empty system has been considered. When a sample is introduced in, say, the lower secondary coil (see Fig.5b) (6) will again give the total voltage drop over the secondaries, but now

$$
\begin{aligned}
& \Delta M=M_{1}\left(1+q_{\mathrm{M}} \chi\right)-M_{1} \\
& \Delta L=L_{1}\left(1+q_{\mathrm{L}} \chi\right)-L_{1}
\end{aligned}
$$

Assuming that the field outside the long cylindrical sample is not disturbed the following estimations for $q_{\mathrm{M}}$ and $q_{\mathrm{L}}$ can be made, if the cylindrical samples are much longer than the secondary coil.

Let $O$ be the cross-section of the coil and $O_{\mathrm{p}}$ that of the sample, $n$ the number of turns of the secondary coil; then

$$
\begin{aligned}
& M_{1}=n \int B \mathrm{~d} O=n B O \\
& M_{2}=n B\left(O+O_{\mathrm{p}} \chi\right)=M_{1}\left(1+\frac{O_{\mathrm{p}}}{O} \chi\right)
\end{aligned}
$$


Then it follows that $q_{\mathrm{M}}=O_{\mathrm{p}} / O$.

(In case the sample is small and not necessarily cylindrical, $q_{M}$ can be written as $f\left(O_{\mathrm{p}} / O\right)$ with $0 \leqslant f \leqslant 1$.)

Likewise $q_{\mathrm{L}}=O_{\mathrm{p}} / O$, since if $B$ is generated by the coil itself, $M$ can be replaced by $L$ in the foregoing.

Equation 6 then reads

$$
\Delta U=j \omega I_{\mathrm{p}} q_{\mathrm{M}} \chi M\left(\frac{1}{N}+\omega^{2} L C\right)
$$

or, making use of (7) and neglecting third powers of $\omega$ and higher,

$$
\Delta U=j \omega L_{\mathrm{p}} q_{\mathrm{M}} \chi M\left[1+2 \omega^{2} L C-j \omega R C\right]
$$

Substitution of (3) gives finally

$$
\begin{aligned}
& \Delta U=j \omega M I q_{\mathrm{M}} \chi\left[1+\omega^{2}\left(L_{\mathrm{p}} C_{\mathrm{p}}+2 L C\right)\right. \\
& \left.-j \omega\left(R_{\mathrm{p}} C_{\mathrm{p}}+R C\right)\right]=j \omega M I q_{\mathrm{M}} \chi A \mathrm{e}^{i \phi}
\end{aligned}
$$

Inserting $q_{\mathrm{L}} / q_{\mathrm{M}}=\beta$ would only change the correction term $2 \omega^{2} L C$ into $(1+\beta) \omega^{2} L C$. It can be seen that the order of magnitude of the correction terms in (10) is correctly estimated. Also, retaining the terms containing $M_{\mathrm{s}}$ in (5a) and (5b) would not alter the result (10) in this approximation, since the correction terms are of the form $\omega^{4} M M_{\mathrm{s}} C^{2}$.

In conclusion we can say that the ratio between $\Delta U / j \omega M I$ and $\chi$ is no longer a real number and depends on $\omega$. The effect of the factor $\mathrm{e}^{i \phi}$ is to produce a rotation from the $\chi$ to the $\Delta U$ direction in the complex plane. Difficulties in the interpretation of the results may therefore arise in such cases when the smallest of the $\chi$ components is of the order of the other multiplied by $\sin \phi$.

A second error, not treated here, arises from the fact that the actual field felt by the sample is produced by $I_{\mathrm{p}}, I_{1}$, and $I_{2}$ together, as can be seen from (3), (5a), and (5b). Including this effect would only introduce more detailed calculations and would not give noticeably different results for those frequencies where the above approximations are valid.

A different arrangement ${ }^{2}$ of secondary coils, consisting of three sections is frequently used. Then the middle section has twice as many turns as the outer sections and is connected with them in series opposition. This middle section might contain the sample to be investigated. The frequency dependence of such a system can be calculated, again neglecting coupling of secondary windings, as follows. The potential drop across each section is

$$
\Delta U_{i}=\frac{j \omega M_{i} I_{\mathrm{p}}}{1-\omega^{2} L_{i} C_{i}+j \omega R_{i} C_{i}}(i=1,2,3)
$$

Let $n$ be the number of turns of an outer secondary coil, then

$$
\begin{array}{ll}
\text { for } L \sim n^{2} & , \quad L_{1}=L_{3}=1 / 4 L_{2} \\
\text { for } M \sim n & , \quad M_{1}=M_{3}=1 / 2 M_{2} \\
\text { for } R \sim n, & R_{1}=R_{3}=1 / 2 R_{2}
\end{array}
$$

The total voltage drop across the system will not now vanish, as was the case in the two-coil-system, which can be seen after insertion of the values in the numerator of (11). The empty-coil effect of such a system is therefore in practice much greater than that used in our measurements.

\section{The adjustment of the PSDs}

As can be seen from Fig. 3 the signal produced by the empty measuring coil system can be compensated by adjusting a variable inductor and a resistor. The condition for equilibrium then reads

$$
\sum_{i}^{j} j \omega M_{i} \mathrm{e}^{j \phi i}+Z_{\mathrm{eff}}=0
$$

where $M_{i}$ and $\phi_{i}$ describe the amplitude and phase of the voltages across the inductive elements and $Z_{\text {eff }}$ compensates for the losses. Actually Hartshorn's design was the first bridge where loss components could be compensated.

As shown in the preceding section the extra induced voltage will be in phase with the bridge current and be proportional to the apparent differential susceptibility. Therefore, to measure the components of $\chi$ it is sufficient to adjust the PSD (and the connected recorders) by means of signals in phase with or perpendicular to the bridge-current.

First we will consider an off-balance signal due to a change of $Z_{\text {eff }}$. The loss compensation part is shown in Fig.6.

The voltage drop $U$ between points 3 and 4 due to a bridge current $I$ flowing from point 1 to 2 is

$$
U_{34}=\frac{Z_{1} Z_{2}}{Z+Z_{1}+Z_{2}} I
$$

A convenient assumption is

$$
\begin{aligned}
& Z_{1}=R_{1}+j \omega l_{1} \\
& Z_{2}=R_{2}+j \omega l_{2} \\
& Z=\frac{R}{1+j \omega R C}
\end{aligned}
$$

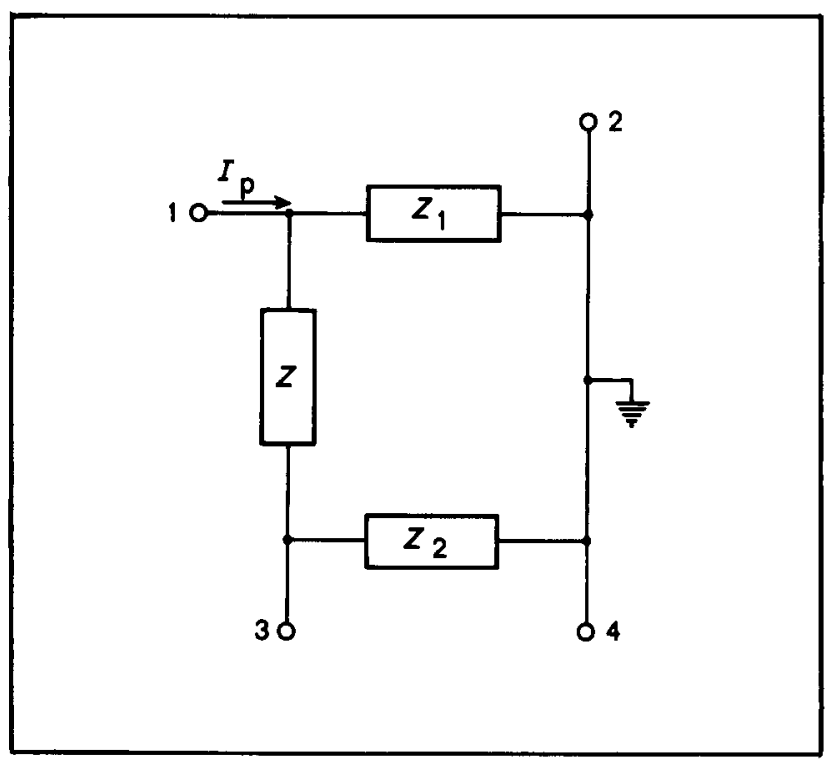

Fig.6 Loss compensation part of the bridge circuit 


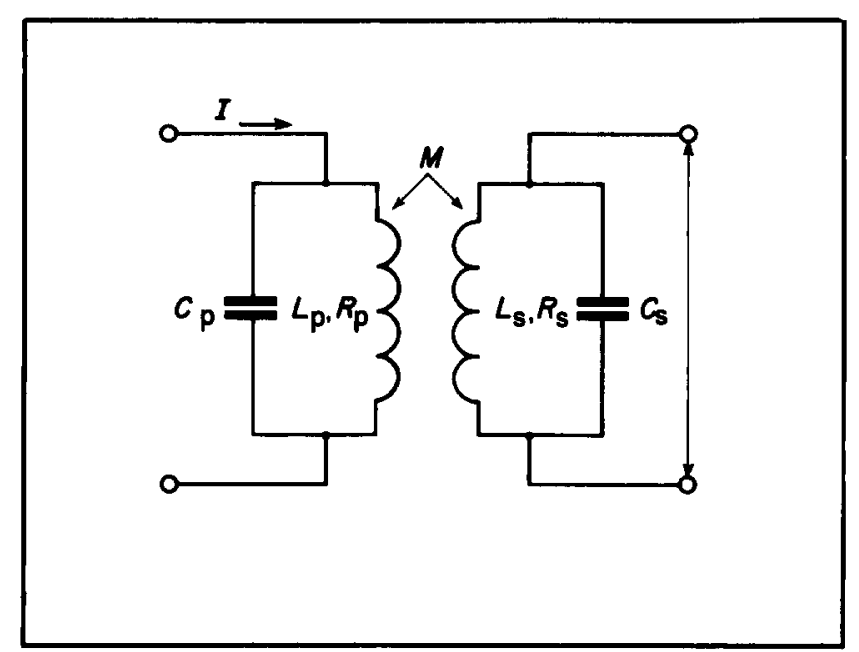

Fig. 7 Simplified circuit of the astatic measuring coil-system

since $R_{1}$ and $R_{2}$ are less than $10 \Omega$ and $R$ is always greater than $20 \Omega l_{1}$ and $l_{2}$ are the internal self inductances of the wires. Rewriting the above equation, neglecting $R_{1}$ and $R_{2}$ in the denominator and assuming $\omega R C<<1$, gives

$$
U_{34}=\left[\frac{R_{1} R_{2}}{R}+j \omega C R_{1} R_{2}+j \omega\left(\frac{R_{2} l_{1}+R_{1} l_{2}}{R}\right)\right] I
$$

The consequence of this equation is that when the bridge is in equilibrium, the reading of the variometer depends on the product $R_{1} R_{2}$ and varies linearly with this value. This could be verified experimentally, showing that the values of $C$ is independent of $R$ and therefore mainly results from the wiring. A second conclusion that the change in the product $R_{1} R_{2}$ at low frequencies, where $l_{1}$ and $l_{2}$ almost vanish, is independent of frequency, can be roughly verified. Lastly we conclude that an off-balance signal produced by the loss circuit cannot be used to adjust precisely the phase sensitive detectors in a direction perpendicular to the bridge current. The question of whether there exists a better signal defining the direction of the $\chi$ components, can be answered by considering the other bridge elements and the value of $\chi$ under special external circumstances.

The relation between the voltage drop $U$ across the secondary coil and the bridge current is given by (see Fig.7)

$$
U=\frac{j \omega M I}{\left(1-\omega^{2} L_{\mathrm{p}} C_{\mathrm{p}}+j \omega R_{\mathrm{p}} C_{\mathrm{p}}\right)\left(1-\omega^{2} L_{\mathrm{s}} C_{\mathrm{s}}+j \omega R_{\mathrm{s}} C_{\mathrm{s}}\right)}
$$

The term describing the induced voltage in the primary circuit due to the secondary current is omitted since the output impedance of the signal source $(82 \Omega)$ is small compared to $1 / j \omega C_{\mathbf{p}}$ at low frequencies. For the variometer it was found that the resonance frequencies of both primary and secondary circuit are much greater than $1 \mathrm{MHz}$, so the assumption, that the variometer acts as a pure mutual inductor at frequencies lower than say $100 \mathrm{kHz}$, is permitted. It should be noted that only the real part of the internal impedance, due to the skin effect of the wiring, in first order, is responsible for a phase shift different from $\pi / 2$. Therefore one method of adjusting the PSDs is provided by the pure behaviour of the variometer at all frequencies of interest. Furthermore, this allows us to calibrate the impurities of the other circuit elements. The second method makes use of the fact that $\chi$ is a real number, that is, $\chi^{\prime \prime}=0$. This happens, for small amplitudes, for paramagnetic salts as well as for superconductors in the absence of a constant field. Then insertion of the sample in the measuring system will produce an off-balance signal in the $\chi^{\prime}$ direction only. So both methods make it possible to adjust the PSDs in the $\chi^{\prime \prime}$ direction, that is the direction where changes of $\chi^{\prime}$ are not observable. Small phase errors may now be introduced by varying in steps the gain of the pre-amplifier and by the fact that one PSD has to be shifted over $90^{\circ}$ to observe the $\chi^{\prime}$ signal. However, these errors can be calibrated if necessary as functions of frequency. Measurements showed that the total error never exceed $1 / 2^{\circ}\left(\sin 1 / 2^{\circ}=9 \times 10^{-3}\right)$. The equivalence of the methods described above can be verified experimentally. This indicates that at frequencies lower than $5 \mathrm{kHz}$ the assumptions made hold and no corrections need be made to the $\chi^{\prime}-\chi^{\prime \prime}$ curves plotted.

\section{The step variable mutual inductor}

The last part of the bridge circuit to be discussed is the step variable mutual inductor. Several devices described in the literature may be briefly mentioned here. Campbell 22 constructed a two-decade mutual inductor using ten-fold stranded wire for the secondary coil to obtain almost equal steps in each decade. The primary and the largest secondary coils are constructed in pairs to obtain a nearly astatic assembly. However, as will be discussed later, the coupling between the secondary coils and their self and intercapacitances introduce large impurities at higher frequencies. At low frequencies (say up to $500 \mathrm{~Hz}$ ) the Campbell inductor is unsurpassed for accuracy and ease of construction and manipulation.

Four-decade versions were published by Casimir et al ${ }^{2}$ and De Klerk and Hudson. ${ }^{3}$ The principal defect of Campbell's inductor arises from the stranded secondaries. The first attempts to reduce these defects involved reducing the number of secondary coils. This, for instance, was done by Butterworth ${ }^{23}$ using three separate coils in each decade, having mutual inductances with the primary of 6,3 , and 1 dial units respectively. However, they must now be independently adjusted. Any number of units between 0 and 10 can be obtained by subtraction and addition of the secondaries. Erickson 4 introduced a four-coil decade system with a 1,2, 2, 5 sequence for the secondaries using an astatic primary pair for the largest five decades and a single primary for the sixth decade, the variometer and correction coils. In this design the concept of internal calibration is also introduced, since in most measurements relative changes in mutual inductance suffice. According to the authors, this bridge has a usable frequency range up to $5 \mathrm{kHz}$. The design adopted for our investigation was that used by De Klerk and Hudson, ${ }^{3}$ making use of tenfold twisted wire for the secondary coils. This guaranteed that the secondary coils are equal, to a few parts in $10^{4}$. For the tenth turn decade an earlier modification was used, consisting of two loops in series-opposition, at $100 \%$ and $90 \%$ of the field produced by the primary coil. The mutual inductance of 1 turn is approximately $3 \mu \mathrm{H}$. The absolute value of the total mutual inductance that can be measured is up to $3.5 \mathrm{mH}$.

We studied the frequency dependence of the bridge in the following way. The assumption that the variometer, because of its high resonant frequencies $(\gg 1 \mathrm{MHz})$, behaves as 
an ideal mutual inductor below $100 \mathrm{kHz}$, allowed us to express the values of the inductance and resistance of the other elements in a frequency dependent number of variometer divisions $(1000 \mathrm{VMD} \sim 1$ turn at low frequency $\sim$ $3 \mu \mathrm{H}$ ). In this way we obtained an 'internal' calibration of the bridge using one element, which had neither inductive nor capacitive coupling with other elements or the surroundings, as a standard.

The experimental results can be summarized as follows.

1. The primary single layer winding has a very low parallel capacity $(60 \mathrm{pF})$ and can be considered as a pure self inductance in the bridge scheme (Fig.8). As a consequence the field is in phase with the bridge current.

2. The lowest resonance frequency of the bridge is that of the largest secondary coil, that is $10 \times 100$ turns. The observed resonance frequency is $8160 \mathrm{~Hz}$. Estimating $L_{10 \times 100}=9 \times 10^{-2} \mathrm{H}$ gives $C=4 \mathrm{nF}$ (approximately) as an effective value for the parallel capacity.

3. The 10 and 1 turn decades have higher resonance frequencies but, due to the magnetic coupling with the $10 \mathrm{x}$ 100 turn coil, they will follow the frequency dependence of the largest secondary coil at higher frequencies.

4. The $1 / 10$ turn system will have a very large frequency dependence since it consists of two coils in series-opposition at $100 \%$ and $90 \%$ of the primary field. The system at $90 \%$ field can be considered to be only weakly coupled with the large secondary coils while the system at $100 \%$ of the field is strongly coupled and will have as a consequence a more pronounced frequency dependence.

It is obvious that the difference of the two induced voltages is increased by the same amount as the 1-turn voltage.

If for example $9 / 10$ turn $=900$ VMD at all frequencies and the 1 turn will increase from $1000 \mathrm{VMD}$ at low frequencies to $1100 \mathrm{VMD}$ at high frequencies, the resulting $1 / 10$ turn will increase from 100 to $200 \mathrm{VMD}$.

So far the influence of the inter-capacities between secondary windings has not been considered. The simplified scheme describing one-half of the astatic step variable inductor is given in Fig.8.

The components of the real and imaginary part of the voltages seen by the secondary circuit relative to $j M I_{\mathrm{p}}$ are

$$
\begin{aligned}
\frac{U}{j \omega M I_{\mathrm{p}}} & =\frac{1-\omega^{2} L C}{\left(1-\omega^{2} L C\right)^{2}+\omega^{2} R^{2} C^{2}} \\
& -\frac{j \omega R C}{\left(1-\omega^{2} L C\right)^{2}+\omega^{2} R^{2} C^{2}} \\
& \sim \frac{1}{1-\omega^{2} L C}-\frac{j \omega R C}{\left(1-\omega^{2} L C\right)^{2}}
\end{aligned}
$$

The $C$ value can also be obtained from the second term and was found to agree reasonably well with the value of $4 \mathrm{nF}$ calculated from the resonance frequency. The inductive component is shown in the lower part of Fig. 9 for the 100 , 10 , and 1 turn decade.

\section{New design for the step-variable inductor}

The effects which are, as discussed above, responsible for the frequency dependence of the inductive bridge elements

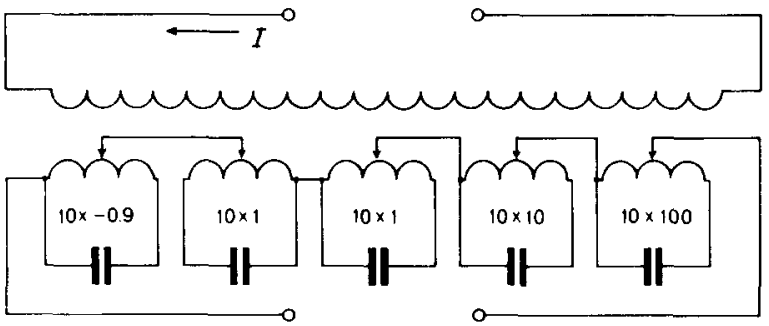

Fig.8 Simplified scheme of the half of the astatic step variable mutual inductor

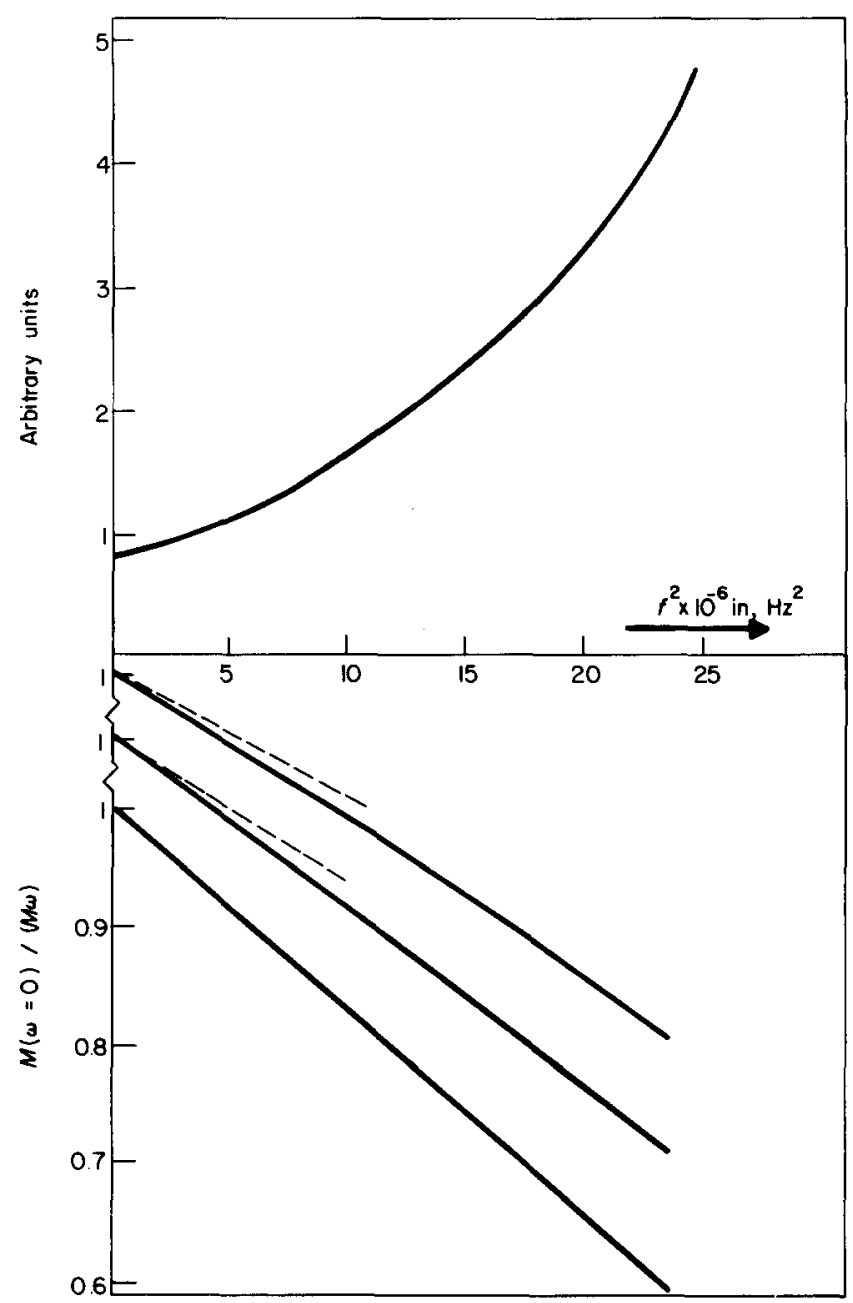

Fig.9 Experimental results for the components of $U$. In the upper half the parabolic behaviour of the $U$-component in phase with the primary current for the 100 turn decade [see (13)] is shown

In the lower part $M(0) / M(\omega)$ is a straight line for the 100 turn decade in agreement with (13). The 10 and 1 turn decades at low frequencies have less steep slopes indicating the lower $L C$ values of these coils. At higher frequencies the coupling with the 100-turn decade changes the slope

can be considerably reduced by using toroidal windings in both primary and secondary circuits.

For mutual inductors of low $M$ value, that is, when the secondary winding still consists of one turn, the advantage gained using the ten-fold twisted wire to get equal decade steps can be retained. By placing the decade switch close to the secondary winding the parasitic capacitances will be very small, about $<100 \mathrm{pF}$, and the frequency dependence will be mainly due to the parallel capacity of the primary winding because of its much greater self inductance. 
Choosing for the primary winding a toroid with square cross-section (side $d$ ) and inner radius $r$ and $N$ turns, the mutual inductance of one secondary loop becomes (at low frequencies)

$$
\begin{aligned}
& M=\frac{\phi}{I}=\frac{\mu_{0} \int H \mathrm{~d} O}{I}=\mu_{0} d \int_{r}^{r+d} \frac{N}{2 \pi r} \mathrm{~d} r \\
& =\frac{\mu_{0} d}{2 \pi} N \ln \frac{r+d}{r} \sim \frac{\mu_{0}}{2 \pi} N \frac{d^{2}}{r} \\
& M(\omega)=\frac{\mu_{0} d}{2 \pi} N \ln \left(\frac{r+d}{r}\right)\left(1-\omega^{2} L_{\mathrm{p}} C_{\mathrm{p}}+j \omega R_{\mathrm{p}} C_{\mathrm{p}}\right)^{-1}
\end{aligned}
$$

This arrangement was substituted for the $1 / 10$ turn decade of the bridge described previously. No impure behaviour of the system was observed up to $5 \mathrm{kHz}$ using the variometer as a standard. Since the geometry of the variometer and the new $1 / 10$ turn decade is quite different, the absence of impurities supports the assumption made already, that the variometer acts as a pure mutual inductor. The second stepvariable inductor constructed, but not used in the experiments, consists of eleven toroidal mutual inductors, with value $M_{i}$ approximately $2^{i} M_{0}$ where $M_{0} \sim 6 \times 10^{-7} \mathrm{H}$. The factors $2^{i}$ are obtained by geometrical considerations, as described below. No attempts have been made to adjust them, but the factors could be easily measured at low frequencies by internal calibration, as well as by comparison with the decade bridge. The sign of the induced voltage, as seen in the secondary circuit for each inductor, is governed by a switch in the primary circuit.

The advantages of this arrangement are various:

1. Toroids approximate best the ideal astatic behaviour required to avoid coupling with the surroundings.

2. The toroids can be built together in such a way that no unwanted magnetic coupling between the various toroids exists.

3. The use of the diadic system provides the smallest number of mutual inductors.

4. The number of toroids necessary for compensation is specified by the maximum signal expected from the measuring coil system. The primaries of all larger toroids can then be switched off. The three largest secondaries can also be switched off. In this way the frequency range at lower signal levels, that is, equivalent signals with $|\Delta M|<$ $100 \mu \mathrm{H}$, can be considerably increased.

5. At a given frequency every signal proportional to $M$ with $|M|<M_{\max }$ can be compensated with the same primary impedance for the signal source and the same input impedance for the detectors.

The frequency range of the bridge is limited now by two factors. First, the lowest resonance frequency of the coils sets a limit, but since this frequency for the step-variable inductor concerned can be far larger than $100 \mathrm{kHz}$, a second limit is set by the loss part of the bridge (consisting of thick wired resistors). The resistors used in our construction behave ohmically up to $30 \mathrm{kHz}$. By measuring off-balance voltages however, as described before, the nonohmic behaviour of the resistors will not influence the results. Therefore the upper limit set to this bridge is of the order of $100 \mathrm{kHz}$.

The toroid bodies were machined from 'perspex' (polymethylmethacrylate). A cross-section is shown in Fig. 10 .

The cross-section of the inner toroid is a regular octagon. Slits on the inner and outer cylindrical faces fix the winding in place. In the upper plane a groove is scored in which a single turn, necessary to compensate the single loop effect of the toroid, can be placed. This toroid is used as the secondary winding. The outer toroid body consists of two halves enclosing the inner toroid; then four sets of splits on the tilted faces are used to fix the winding. The inner windings are wound from $40 \times 0.03 \mathrm{~mm}$ litz wire, the outer ones from $0.5 \mathrm{~mm}$ copper wire.

The dimensions and number of turns together with other significant properties are given in Table 1 .

Table 1. Values for the characteristic properties of the toroids

\begin{tabular}{llllllllll}
\hline $\begin{array}{l}\text { Toroid } \\
\text { No }\end{array}$ & $\begin{array}{l}r_{1} \\
\mathrm{~mm}\end{array}$ & $\begin{array}{l}d_{1}, \\
\mathrm{~mm}\end{array}$ & $\begin{array}{l}d_{2,} \\
\mathrm{~mm}\end{array}$ & $N_{1}$ & $N_{2}$ & $\begin{array}{l}L_{\mathrm{p}}, \\
\mu \mathrm{H}\end{array}$ & $\begin{array}{l}L_{s} \\
\mu \mathrm{H}\end{array}$ & $\begin{array}{l}C_{12}, \\
\mathrm{pF}\end{array}$ & $\begin{array}{l}\mu \mathrm{H} \\
\mu \mathrm{H}\end{array}$ \\
\hline 1 & 30 & 20 & 5 & 90 & 45 & 17.9 & 0.28 & 17 & 0.56 \\
2 & 30 & 20 & 5 & 90 & 90 & 17.9 & 1.12 & 17 & 1.12 \\
3 & 30 & 20 & 10 & 90 & 45 & 17.9 & 1.12 & 34 & 2.24 \\
4 & 30 & 20 & 10 & 90 & 90 & 17.9 & 4.47 & 34 & 4.47 \\
5 & 60 & 40 & 20 & 180 & 45 & 143 & 2.24 & 67 & 8.95 \\
6 & 60 & 40 & 20 & 180 & 90 & 143 & 8.95 & 67 & 17.9 \\
7 & 60 & 40 & 20 & 180 & 180 & 143 & 35.8 & 67 & 35.8 \\
8 & 120 & 80 & 40 & 360 & 90 & 1145 & 17.9 & 135 & 71.6 \\
9 & 120 & 80 & 40 & 360 & 180 & 1145 & 71.6 & 135 & 143 \\
10 & 120 & 80 & 40 & 360 & 360 & 1145 & 286 & 135 & 286 \\
\hline
\end{tabular}

The mutual inductance can be approximated as follows. Consider the field inside the secondary toroid as constant and equal to that at the centre of the octagon then

$$
H=\frac{N_{1} I}{2 \pi r}
$$

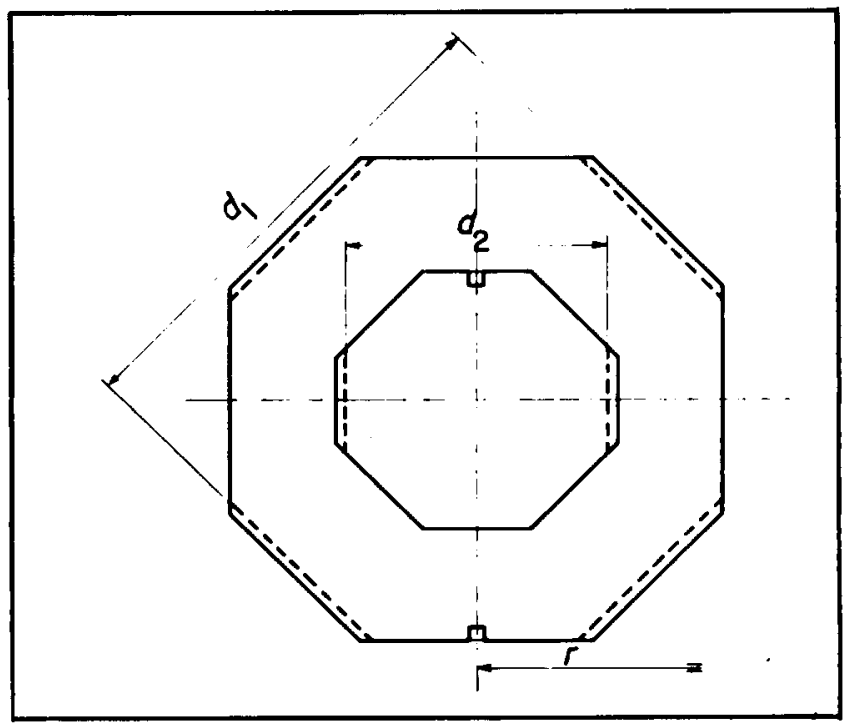

Fig.10 Cross-section of the toroid body 
and

$$
\begin{aligned}
M & =\mu_{0} N_{1} N_{2} \frac{d_{2}^{2}}{r} \frac{(\sqrt{ } 2-1)}{\pi} \\
& =1.66 N_{1} N_{2} \frac{d_{2}^{2}}{r}, 10^{-10} \mathrm{H}
\end{aligned}
$$

where $d_{2}$ and $r$ are expressed in mm. Note that $N_{1} / r$ is a constant.

The actual mutual inductance is greater than that calculated with (14), as would be expected. Toroid No 6 was incorporated in the system twice to measure the frequency dependence of the larger toroids by means of internal calibration. The step size of the smallest inductor is still larger than the maximum obtainable with the variometer. Therefore one toroid with three switchable secondaries of 1,2 , and 4 turns was added to the step variable inductor. The mutual inductance of 1 turn is $10^{-7} \mathrm{H}$.

\section{Coupling of the measuring coil system with the co-axial superconducting magnet}

In the past ten years, when strong field superconducting coils became available, measuring coil systems for the in vestigation of paramagnetic properties of salts and inductive resistance measurements of normal and superconducting metals have frequently been surrounded by superconducting magnets for the production of a dc field.

This may influence the measurements and therefore corrections, due to the interaction of the measuring system and the superconducting coil, have to be considered.

We stated 24 that this interaction can be reduced by a special arrangement of the complete coil system, but within the prescribed length of that paper our statements could not be justified completely. The properties of our self-made superconducting coil magnet are the following: bore $60 \mathrm{~mm}$, outside diameter $92 \mathrm{~mm}$, length $110 \mathrm{~mm}$, inside notch type, maximum field $30 \mathrm{kOe}$ at $80 \mathrm{~A}$ and $T=4.2 \mathrm{~K}$. The coil was wound from $1 \mathrm{~km}$ Niomax S 25/50, homogeneity better than $1: 10^{-4}$ in a sphere with radius $15 \mathrm{~mm}$ at the centre.

Now, when a current $I \mathrm{e}^{-j \omega t}$ is admitted to the primary coil, ac voltages are induced in the secondary coils and the superconducting coil proportional to their mutual inductances with the total primary coil system, $M_{2,1}$ and $M_{\mathrm{S}, 1}$ respectively:

$$
\begin{aligned}
& U_{2,1}=j \omega M_{2,1} I \\
& U_{\mathrm{S}, 1}=j \omega M_{\mathrm{S}, 1} I
\end{aligned}
$$

In our system the superconducting coil (self inductance $L$, resistance leads, and ampère-meter $R_{2}$ ) is short-circuited by a resistance $R_{1}$, to protect the power supply and give an almost constant load to the voltage $U_{\mathrm{S}, 1}$. Therefore an ac current $I_{\mathrm{S}}$ may flow in the superconducting coil

$$
I_{\mathrm{S}}=\frac{j \omega M_{\mathrm{S}, 1} I}{j \omega L+R_{2}+R_{1}}
$$

Even at frequencies as low as $2 \mathrm{~Hz}, R_{1}$ and $R_{2}$ are small enough to be omitted without violating our simple model. So we may put

$$
I_{\mathrm{S}}=\frac{M_{\mathrm{S}, 1}}{L} I
$$

and hence an extra voltage will be induced in the secondary system proportional to the mutual inductance between the superconducting coil and the secondary system

$$
U_{2, \mathrm{~S}}=j \omega \frac{M_{2, \mathrm{~S}} M_{\mathrm{S}, 1}}{L} I
$$

So the total induced voltage becomes

$$
U_{\mathrm{tot}}=U_{2,1}+U_{2, \mathrm{~S}}=j \omega M_{2,1} I\left(1+\frac{M_{2, \mathrm{~S}} M_{\mathrm{S}, 1}}{M_{2,1} L}\right)
$$

The last term, in the brackets, now gives the required correction. It can be made small enough by two methods.

1. Minimizing $M_{\mathrm{S}, 1}$ by choosing the correct number of terms on the two primary coils.

2. Minimizing $M_{2, \mathrm{~S}}$ by shifting the secondary coils along the axis of the superconducting coil.

Since the field of $\mathrm{S}$ is homogeneous $M_{\mathrm{S}, 1}$ is not much affected by such a shift.

In this way $M_{2, \mathrm{~S}} / M_{2,1}$ can be made smaller than an arbitrary constant, say ten, and in our case $M_{\mathrm{S}, 1} / L$ could be made as small as $10^{-5}$ in the sample's absence. The dependence of the correction term on the various mutual inductances and its independence of frequency can be verified experimentally.

It should be noted however, that the absence of corrections with no sample present does not imply the absence of corrections in the presence of the sample since $M_{2}, M_{2,1}$, and $M_{\mathrm{S}, 1}$ all change when the specimen is placed into one of the secondary coils.

1. $M_{\mathrm{S}, 1}$ will be increased due to the finite length of primary coils having different diameters. Also at higher dc fields $M_{\mathrm{S}, 1}$ may change in a minor way, since the shape of the field produced by the coil $\mathrm{S}$ depends on the current flowing through it and its actual ac behaviour at high fields.

2. $M_{2, \mathrm{~s}}, M$, and $M_{2,1}$ may change with frequency and dc field depending on the nature of the specimen.

Then we have in the case where the sample is transparent to the ac field, the correction is negligible as it was with the empty coils, but if the sample excludes the field completely (superconductor, skin effect at high frequencies) or in zero field paramagnetic behaviour $M_{2, \mathrm{~s}} / M_{2,1}$ tends to a ratio of number of turns per metre of the coil $S$ to the effective number of turns per metre of the primary coil system when the sample is large enough.

In our case

$$
\left|\frac{M_{\mathrm{S}, 1}}{L}\right|<3 \times 10^{-4}
$$

always applies while the ratio mentioned above is three. 
Therefore in our coil system the correction term never exceeds 1 in the whole range from 1 to $5 \times 10^{3} \mathrm{~Hz}$ and for $\mathrm{dc}$ fields up to $25 \mathrm{kOe}$.

\section{Part 2. Frequency multiplier with programmable multiplication factor}

\section{Choice of method}

This section describes the tracking frequency multiplier which is used to measure the harmonic content of a distorted sine wave, with the help of a lock-in amplifier. The amplifier can be synchronized externally by a square wave of the same frequency as the harmonic of interest. This frequency is not directly available: it has to be created by a frequency multiplier which converts a square wave with frequency $F$ into a square wave with frequency $N F$. The frequency range of interest and the desired accuracy of the measurements led to a number of requirements:

The conversation factor $N$ programmable from, for example, $1-7 N$ must be an integer with only small variations caused, for example, by jitter.

The frequency range of the output signal must be between 0.2 and $20 \mathrm{kHz}$.

The displacement between the negative zero crossing of the input square wave $F$ and the nearest zero crossing of the output square wave $N F$ must be less than $1^{\circ}$ of the output square wave.

The frequency multiplication method using a phase-lock loop appears less suitable according to the requirements stated.

An investigation of an alternative method, which does not have the disadvantages of the phase-locked loop circuitry, led to the development of a digital conversion technique.

\section{General review: block diagram}

A clock frequency is generated and one binary counter counts the frequency $F_{\mathrm{c}} / 2 N$. At the end of each period of the first harmonic $F$, a transfer pulse $T_{\mathrm{p}_{1}}$ and (somewhat later) a reset pulse $R_{p_{\mathbf{4}}}$ is generated by a timing circuit Ct 1 .

The transfer pulse causes the counter to dump its content into the memory M1.

The reset pulse also initiates the start of a new counting cycle $\mathrm{Cc1}$, the duration of which is equal to period $T$ of the first harmonic $F$.

A second binary counter counts the clock frequency $F_{\mathrm{c}}$. Its content is compared with the content of a second memory M2 by comparator circuitry. As soon as both contents are equal, a reset pulse $R_{\mathrm{p}_{2}}$ and a transfer pulse $T_{\mathrm{p}_{2}}$ are generated by the timing circuit $\mathrm{Ct} 2$. The reset pulse causes the resetting of the counter and the start of a new counting cycle $\mathrm{Cc} 2$, the duration of which is $T_{0} \mathrm{~s}$. The transfer pulse causes the transfer of the number content of the first memory to the second memory M2.

After two-scaling of the reset pulse signal $R_{\mathrm{p} 2}$, a $50 \%$ duty cycle square wave is obtained with frequency $N / T$.

A synchronization circuitry $\mathrm{Cs} 1$ controls the time displacement between the negative zero crossing of $F$ and the nearest negative zero crossing of $N F$, by adding a sinc pulse to the

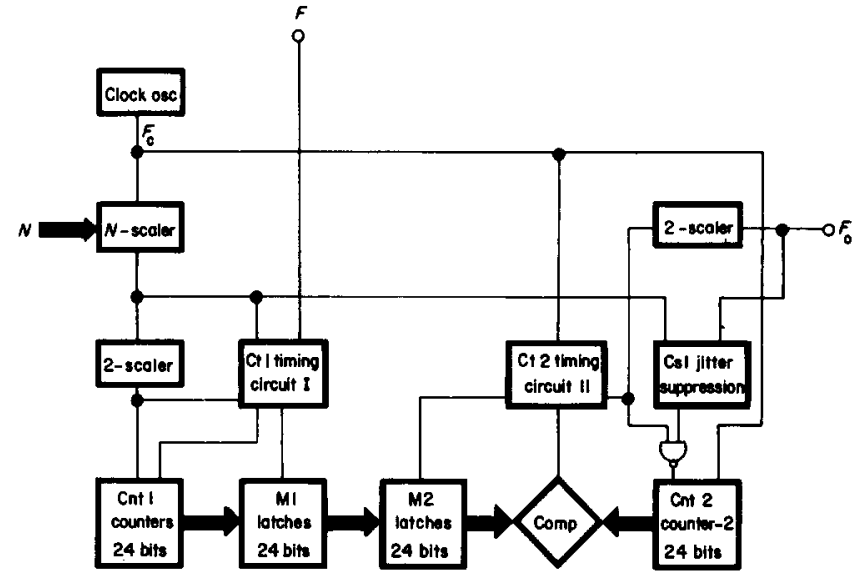

$\mathbf{a}$

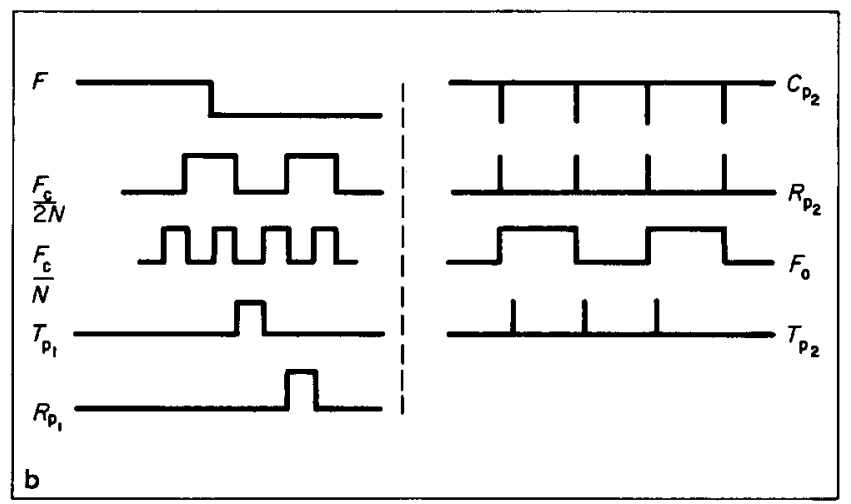

Fig.11 a - Block-diagram of the digital frequency multiplier; b - Time sequency diagram

$F$ - input square wave; $F_{\mathrm{c}}=$ clock signal; $T_{\mathrm{p}_{1}}-$ transfer pulse of first memory; $\boldsymbol{R}_{\mathrm{p}_{1}}$ - reset pulse of first counter $\operatorname{Cnt} 1 ; C_{\mathrm{p}_{2}}$ - output pulse of comparator; $\boldsymbol{R}_{\mathrm{p}_{2}}$ - reset pulse of second counter Cnt 2; $T_{\mathrm{p}_{2}}$ - transfer pulse of second memory

reset of the second counter. The block diagram of this digital frequency multiplier is given in Fig.11a and the time diagram in Fig.11b.

\section{Frequency and jitter behaviour}

Generally the period time $T$ of the first harmonic $F$ is not divisible by the period of the count frequency $F / N$.

Suppose $T$ is divisible by the clock period $1 / F_{\mathrm{c}}$ then $T$ can be written as

$$
T=K \frac{N}{F_{\mathrm{c}}}+\frac{p}{F_{\mathrm{c}}} ; \quad p \text { is integer and } 0<p<N
$$

The duration of $N$ periods $F$ is divisible by $N / F_{\mathrm{c}}$, so the mean content $\bar{I}$ of the memory is constant over each interval of $N$ periods. When the following requirements are met, the mean content per period $T$, taken for $N$ periods $F$, will be equal to $K+p / N$.

Each count pulse $F_{\mathrm{c}} / N$ is counted once and only once.

The content of the memory is equal to the total number of pulses counted by the first binary counter in the preceding period $T$.

After each period $T$ the content of the counter is transferred error free to the first memory.

As a result of the rest term $p / F_{c}$, the content of the memory will be set to $K$ and the rest term will be accumulated. 
As soon as the accumulated rest term is $>N$, then the memory will be set to $K+1$ and the number $N$ is subtracted from the accumulated rest term. At the end of each of $x$ successive periods of signal $F$, the memory is set to $K$, and at the end of the $(x+1)$ th period to $K+1$. The number $x$ follows from the equation $x \leqslant N / p ; x$ is the nearest integer to $N / p$.

The accumulated error is after $(x+1)$ periods of signal $F$ equal to

$$
e=(x+1) \frac{p}{F_{\mathrm{c}}}-\frac{N}{F_{\mathrm{c}}}=\frac{1}{F_{\mathrm{c}}}[(x+1) p-N]
$$

It can be shown that the accumulated error will always be $\left\langle N / F_{\mathrm{c}}\right.$. From the behaviour of the memory content as a function of the period number of signal $F$, frequency and jitter behaviour can be simply derived. The memory content $K$ will cause an output frequency $F_{0}^{\prime}>\bar{F}_{0}=N F$. The difference between the output frequency $F_{0}^{\prime}$ and the mean $\bar{F}_{0}$ is $\Delta F_{\mathrm{H}}$. The memory content $(K+1)$ will cause an output frequency $F_{0}^{\prime \prime}<\bar{F}_{0}=N F$, the difference being $\Delta F_{\mathrm{L}}$.

$$
\bar{F}_{0}=\frac{N F_{\mathrm{c}}}{N K+p} \approx F_{0}^{\prime}\left(1-\frac{p}{N K}\right)
$$

From this

$$
F_{0}^{\prime} \approx \bar{F}\left(1+\frac{p}{K N}\right)
$$

and

$$
\frac{\Delta F_{\mathrm{L}}}{\bar{F}_{0}} \approx \frac{N-p}{N} \frac{\bar{F}_{0}}{F_{\mathrm{c}}}
$$

An expression for $\Delta F_{\mathrm{H}} / \bar{F}_{0}$ can be derived in the same way

$$
\frac{\Delta F_{\mathrm{H}}}{\bar{F}_{0}}=\frac{p}{N} \frac{\bar{F}_{0}}{F_{\mathrm{c}}}
$$

The output frequency remains $F_{0}^{\prime}$ during $X$ periods $F$.

The time difference between the duration of $x N$ periods $F_{0}^{\prime}$ and the duration of $x N$ periods $\bar{F}_{0}$ equals

$$
\Delta t=x N \frac{K}{F_{\mathrm{c}}}-x N \frac{N K+p}{N F_{\mathrm{c}}}=-\frac{x p}{F_{\mathrm{c}}}
$$

The maximum value of this difference is $-X_{\max p} / F_{\mathrm{c}}$ and with $X \leqslant N / p$, it follows

$$
|\Delta t|<\frac{N}{p} \frac{p}{F_{\mathrm{c}}}=\frac{N}{F_{\mathrm{c}}}=N T_{\mathrm{c}}
$$

Thus the phase jitter remains below $2 \pi N T_{\mathrm{c}} / \bar{T}_{0}$.

In Fig.12 the relative frequency variation $\Delta F / \bar{F}_{0}$ for $p=1$, $N=3$ has been drawn.

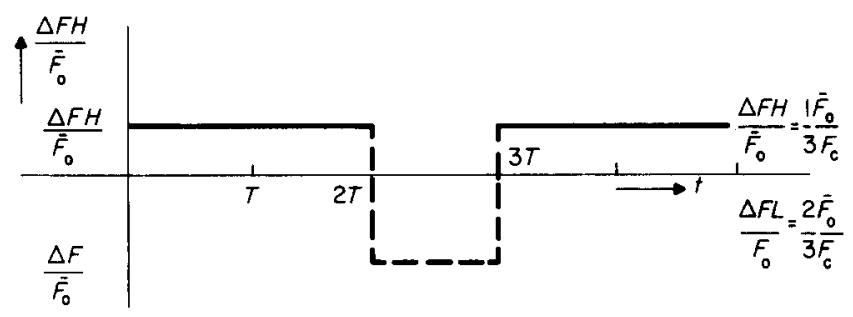

Fig.12 Frequency variation of output square wave $\bar{F}_{0}=N F$, with $p / N=1 / 3$

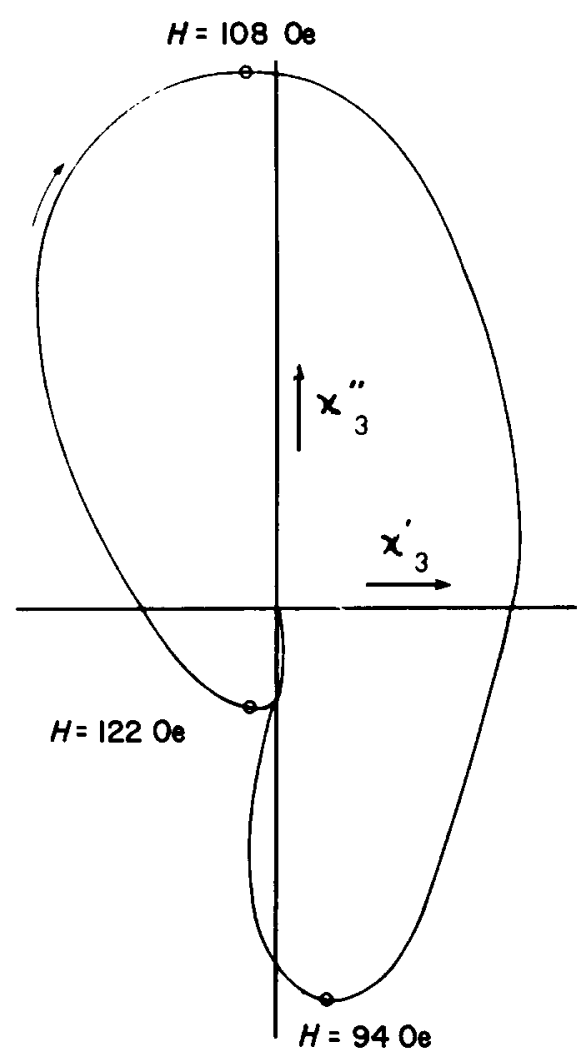

Fig.13 $\chi_{3}^{\prime \prime}$ versus $\chi_{3}^{\prime}$, in arbitrary units, as a function of $H$ of polycrystalline tin

$T=3.11 \mathrm{~K}, \nu=130 \mathrm{~Hz}, h=22 \mathrm{Oe}$

\section{Evaluation}

In the present digital frequency conversion technique the jitter increases proportionally to the multiplication factor $N$.

Another factor is that the mean of the output frequency is exactly $N F$, when this mean is taken over $N$ periods of $F$.

These two properties are not disadvantageous with regard to the multiplication accuracy required, as $N$ is not too large and the variations of $N$ and of the input frequency $F$ are not too rapid.

Further development has resulted in an improvement in performance of several parts of the frequency multiplication system, and the following specifications are attained:

The multiplication factor $N$ is programmable from 1 to 100 . 
The jitter of the output signal $<500 \mathrm{~ns}$, independent of the multiplication factor $N$.

The output frequency $<100 \mathrm{kHz}$.

Evaluation of the present system will make it possible to transform the output signal, a digital triangular voltage of the right frequency, into a digital sine wave of the same frequency, with programmable phase in steps of $1^{\circ}$ at frequencies below $500 \mathrm{~Hz}$, and in steps of $10^{\circ}$ at higher frequencies.

\section{Part 3. Experimental results}

\section{Results for certain superconductors}

Using the equipment, described in the preceding section, experiments have been performed on a number of metals in the normal and the superconducting states. As the results of these experiments will be published separately we will restrict ourselves to presentating certain examples, which illustrate the possibilities of the equipment.

\section{Fourier analysis of ac response}

Type I superconductivity. For a polycrystalline tin cylinder the $x$-values depend strongly on the frequency, the amplitude $h$, the value and the direction of the change of the external field $H$. In Fig. 13 the third harmonic $\chi_{3}$ is given as a $\chi_{3}^{\prime \prime}$ versus $\chi_{3}^{\prime}$ curve for decreasing $H$. The parameter along the curve is the external field.

Type II superconductivity. The $\chi_{5}^{\prime \prime}$ versus $\chi_{5}$ curve of a pure niobium cylindrical single crystal is plotted in Fig.14.

The shape of the curve depends on frequency, amplitude of the alternating field, and only on the value of the external field. The parameter along the curve is the external field. Between the origin and the turning point at $H_{\mathrm{p}}$, the ac field penetrates slightly into the sample, probably because of flux pinning. The field $H_{\mathrm{p}}$ has been defined in a previous paper. 24

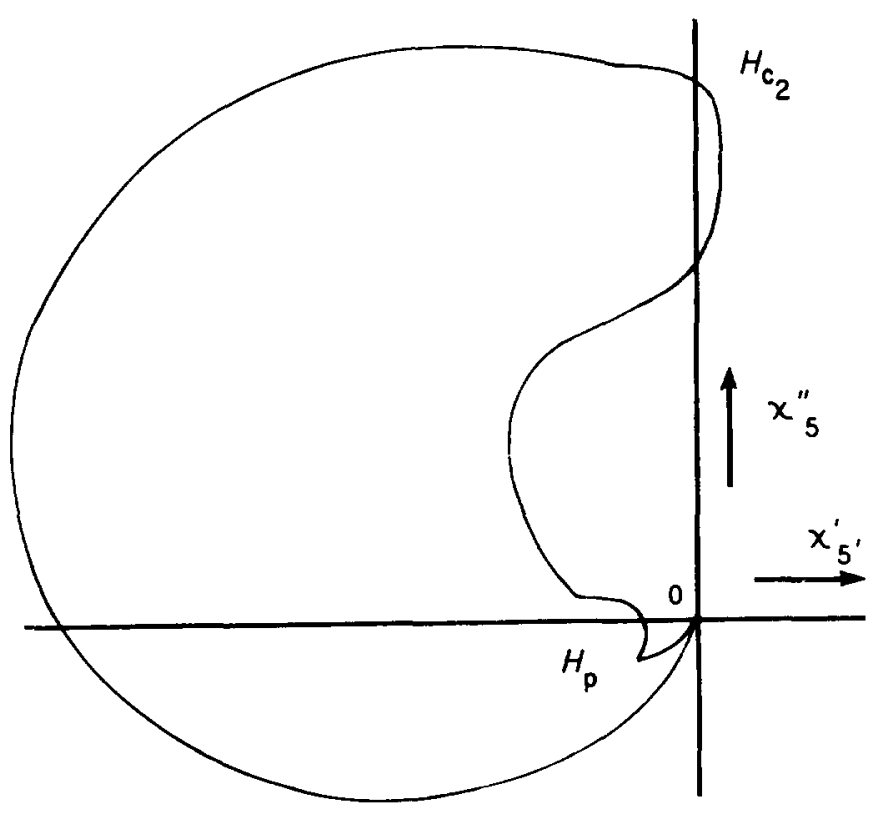

Fig.14 $\chi_{5}^{\prime \prime}$ versus $\chi_{5}^{\prime}$, in arbitrary units, as a function of $H$ of a single crystal of niobium

$T=4.2 \mathrm{~K}, \nu=130 \mathrm{~Hz}, h=22 \mathrm{Oe}$

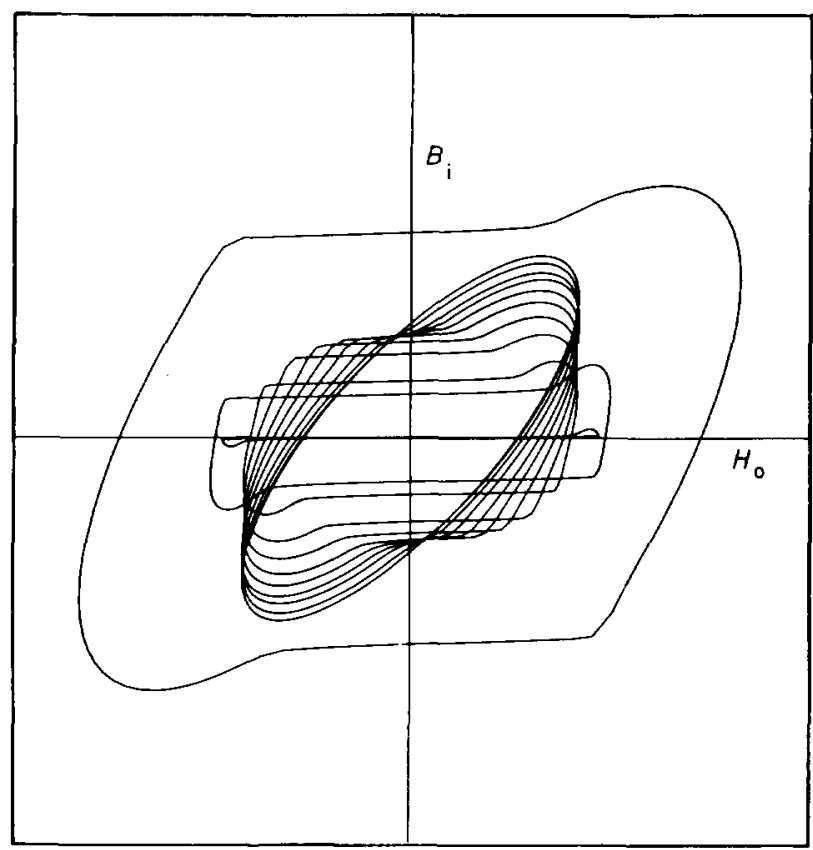

Fig.15 Set hysteresis loops of a solid tin cylinder at decreasing temperature. At the lowest temperature $(1.4 \mathrm{~K})$ a few loops with increasing amplitude are shown; $\nu=130 \mathrm{~Hz}$

Between $H_{\mathrm{p}}$ and $H_{\mathrm{c}_{2}}$ the flux pinning disappears and between $H_{c_{2}}$ and the origin again $\left(H_{\mathbf{c}_{3}}\right)$ the curve shows the influence of surface superconductivity.

In contrast to the model of Rollins and Silcox 12 even harmonics could also be detected. For the odd harmonics the curve differs from the shape predicted by these authors, but tends for $H$ values above $H_{c_{2}}$, to their predicted shape if the frequency and the amplitude of the alternating field are both decreased.

\section{AD converted signal registration}

Hysteresis loops of superconductors. Using the signal sampling equipment, described in part 1 , the signal of the pick-up coil is fed into a computer. By straight-forward calculation the shape of the hysteresis loop can be obtained.

A typical set of hysteresis loops for a long tin cylinder is shown in Fig.15. The amplitude is taken as somewhat smaller than the critical field at zero temperature. These large amplitude ac fields are produced by superconducting coils. $^{27}$

$E-J_{e}$ relations. In previous papers 26,27 the response of hollow cylinders of pure niobium is presented. The signal sampling technique allows quantitative determination of $E-J_{\mathrm{e}}$ (or $J_{\mathrm{n}}-J_{\mathrm{s}}$ ) curves to be quantitatively determined in the time dependent regime. $J_{\mathrm{e}}$ is then the superconducting component of the total screening current in the thinwalled cylinder (see Fig.16).

The authors are greatly indebted to Mr K. Bouwknegt for his assistance in building the frequency multiplier and to $\mathrm{Mr}$ J. A. Ulfman and Mr H. A. Steffens for constructing the bridges and for technical assistance during the measurements and to Miss E. de Boer for typing the manuscript. Enlightening discussions with Prof Ir D. Bosman,

Prof Dr B. S. Blaisse, Prof Ir H. G. de Winter, Dr G. J. C. Bots, Ir L. P. J. Veelenturf, Dr D. de Klerk and Dr D. Grant are also gratefully acknowledged. 


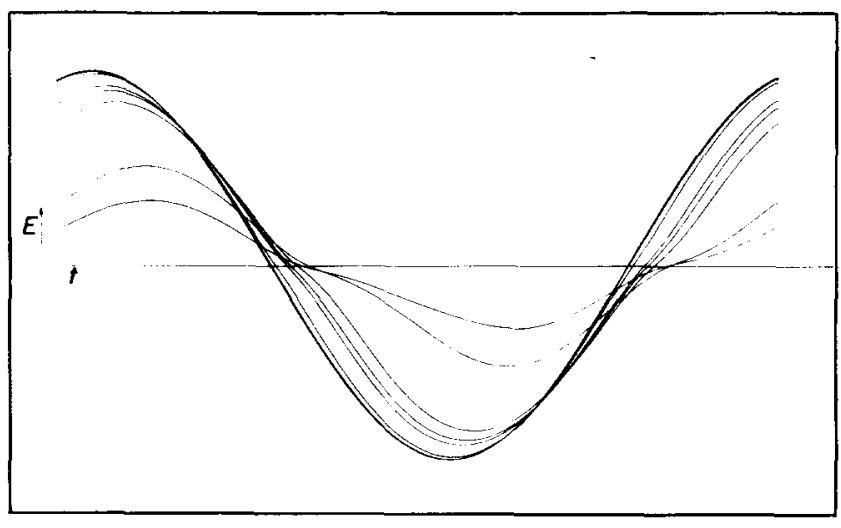

a

Fig.16 a - The time dependence of $E$ in a thin walled niobium cylinder at static field values between $H_{\mathrm{c}_{2}}$ and $H_{\mathrm{c}_{3}}$

$b-$ Corresponding $J_{e}$ versus $J_{n}$ loops. $J_{e}$ is the excess current (superconducting component) of the total screening current; $J_{n}$ is the normal component and equals $a E$

\section{References}

1 Verstelle, J. C., Curtis, D. A. Handbuch der Physik Vol 18, (Springer - Verlag, Berlin, 1968)

2 Casimir, H. B. G., De Haas, W. J., De Klerk, D. Physica 6 (1939) 241 (see also: De Klerk, D., Thesis, Leiden 1948) De Klerk, D. Hudson, R. P. J Res NBS 53 (1954) 173

4 Erickson, R. A., Roberts, L. D., Dabbs, J. W. T. Rev Sci Instr 25 (1954) 1178

5 Wiebes, J., Hulscher, W. S., Kramers, H. C. Appl Sci Res $11 B(1964) 213$

6 Pillinger, W. L., Jastram, P. S., Daunt, J. G. Rev Sci Instr 29 (1958) 159

7 Hoffman, J. T., Sapp, R. C. J Appl Phys 39 (1968) 837

8 Van Dijk, H. Temperature, Its Measurements and Control in Science and Industry, Vol 2 (Reinhold Publ Corporation, New York, 1955) 199; Comm Kamerlingh Onnes Lab, Suppl No $112 \mathrm{c}$

9 Cremer, R., Gabrielsen, N. Hüfner, S., Nicolay, K., Weber, G. Phys Kondens Materie 5 (1960) 5

10 De Vries, A. J., Livius, L. W. M. Appl Sci Res 17 (1967) 31

Zenneck, J. Ann der Phys IV 9 (1902) 497

Rollins, R. W., Silcox, J. Phys Rev 155 (1967) 404

Van der Klein, C. A. M., Elen, J. D., Wolf, R., De Klerk, D. Physica 49 (1970) 98

14 De Klerk, D., Van der Klein, C. A. M. Comm Kamerlingh Onnes Lab, No 388 a; J Low Temp Phys 6 (1972) 1

15 Van Engelen, P. P. J., Bots, G. J. C., Blaisse, B. S. Phys Lett 19 (1965) 465

16 Hague, B., Foord, T. R. Alternating Current Bridge Methods, 6 th edn (Pitman Publishing, 1971)

17 Pedersen, P. Electrician 83 (1919) 523

18 Hartshorn, L. J Sci lnstr 2 (1925) 145

19 Campbell, A. (see reference 16, p 186)

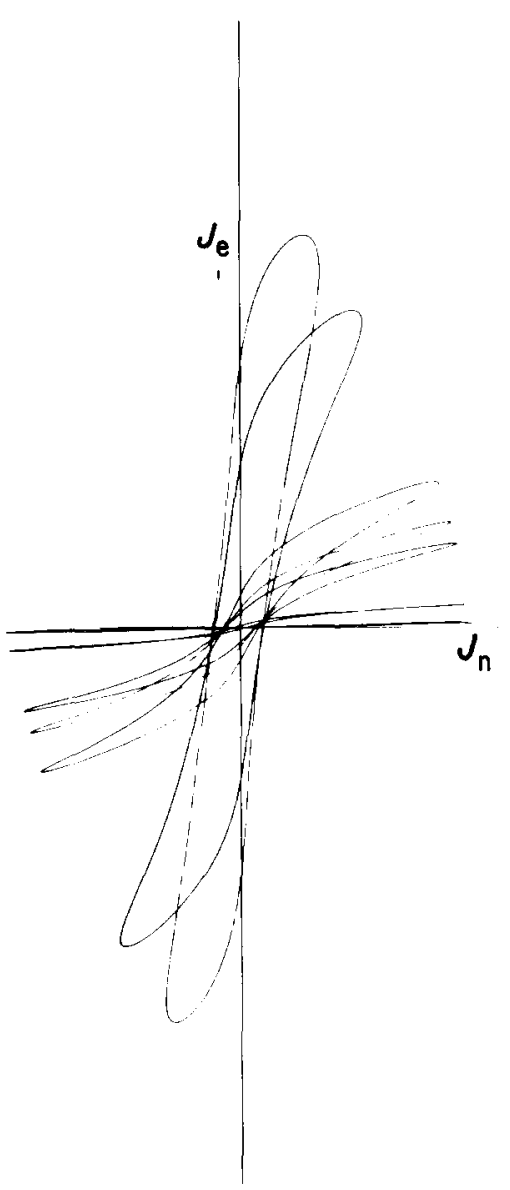

b

Butterworth, S. Proc Phys Soc 33 (1921) 312

Hartshorn, L. Proc Phys Soc 38 (1926) 302

Campbell, A. Phil Mag 6 (1908) 155 and Proc Phys Soc 21 (1907) 69

23 See reference $16, p 176$

24 Van de Klundert, L. J. M., Alberga, G. E., Van der Marel, L. C., Leynse, C. Proc ICEC3 (Berlin 1970) 346

25 Gardner, F. M. Phase Lock Techniques (Wiley, New York, 1966)

26 Van de Klundert, L. J. M., Gijsbertse, E. A., Van der Marel, L. C. Physica 69 (1973) 159

27 Van de Klundert, L. J. M. 'Frequency and amplitude dependence of hysteresis loops of solid and hollow superconducting cylinders', Proc International Meeting on Flux Pinning, Sonnenberg, September 1974 